\title{
Irregular Polyomino-Shaped Subarrays for Space-Based Active Arrays
}

\author{
R. J. Mailloux, ${ }^{1,2}$ S. G. Santarelli, ${ }^{2}$ T. M. Roberts, ${ }^{2}$ and D. Luu ${ }^{2}$ \\ ${ }^{1}$ Electrical and Computer Engineering Department, University of Massachusetts, Amherst MA, 01003, USA \\ ${ }^{2}$ Sensors Directorate, Electromagnetic Technology Division, Antenna Technology Branch, \\ Air force Research Laboratory, MA, 01731, USA
}

Correspondence should be addressed to R. J. Mailloux, robert.mailloux@hanscom.af.mil

Received 27 October 2008; Accepted 22 January 2009

Recommended by Stefano Selleri

This paper presents new results showing the application of polyomino-based subarrays to limited field of view and wideband, wide-angle scanning. This technology can reduce the number of phase controls in arrays used for limited sector coverage or the number of time delay devices for wideband radar or communications, and so can reduce the cost of space-based active arrays. We concentrate on the wideband application. Results are presented by comparing the gain and peak sidelobe results of irregular polyomino subarray-based arrays with those of rectangular subarrays. It is shown that using irregular polyomino subarrays can result in a major decrease in sidelobes while presenting, in most cases, only a few tenths of a dB gain reduction compared to rectangular subarrays.

Copyright ( 2009 R. J. Mailloux et al. This is an open access article distributed under the Creative Commons Attribution License, which permits unrestricted use, distribution, and reproduction in any medium, provided the original work is properly cited.

\section{Introduction}

The increasing use of large scanning arrays for space applications emphasizes a growing need for subarray technology to reduce the number of controls in systems that scan over limited fields of view (LFOV) or to minimize the number of time delays for arrays with active phase control at the element level. These two applications are addressed by the same subarray technology, and so these will be discussed together initially before presenting detailed results for the wideband case. Subarray configurations for the two applications are illustrated in Figure 1.

LFOV applications include synchronous satellites, where the earth subtends a conical angle of $8.7^{\circ}$ half angle. Other satellite systems sometimes scan a high-directivity beam over even smaller angles to do detailed mapping. In the LFOV configuration shown at left in Figure 1, there is no phase shift at the element level, but phases are introduced at the input port to each subarray. The subarray is used as an oversized element and the gain and pointing accuracy is maintained by phase control at the subarray input ports. In the time-delayed configuration of Figure 1, the subarray has phase shifters at the element level and time delays at each subarray input [1].
The phase shifters scan the subarray beam direction, but the time delays are required to keep the array pattern stationary for all frequencies within the frequency band.

The subarrays most commonly used for either application are rectangular groups of elements. When placed in a periodic array these radiate discrete sidelobes called quantization lobes at the location of the grating lobes of the array factor.

Solid-state modules with phase shift pattern control are now being used in many space applications, and they can be designed to have good performance over wide bandwidths but the phenomenon of beam "squint" limits their use operationally. Beam squint is the property of a phase steered array that results in frequency-dependent pointing angles. This can result in severely limited instantaneous bandwidth for large phase-steered arrays. Time-delay-steered arrays do not exhibit this phenomenon.

Time delay control of scanning arrays remains an expensive and often impractical option, because of the difficulty of implementing long time delays at the element level or switched time delay tree networks. Digital or optical beam forming networks can introduce time delay at element or subarray level but for reasons of economy it 


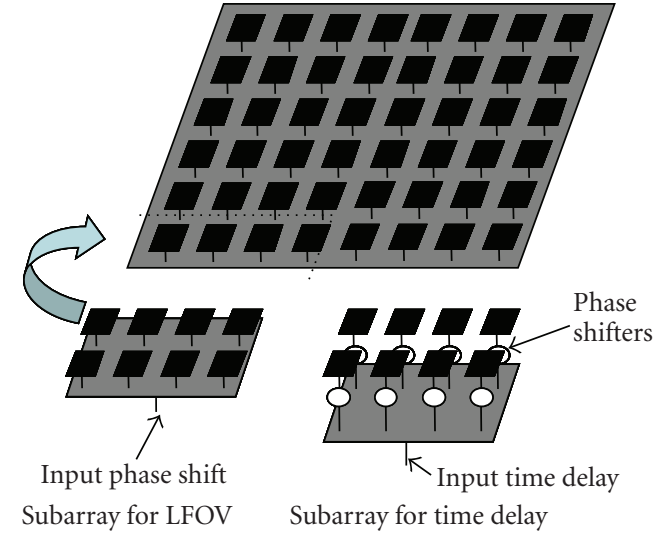

FIgURE 1: Subarray applications to LFOV and time-delayed wideband arrays.

is often convenient to introduce time delay at the subarray level. New and projected applications with requirements for bandwidths up to limits of 10:1 place increasingly severe restrictions on the size of phase steered arrays or on the subarray technology that enables the use of relatively few time delay networks.

The two cited applications for subarray technology exhibit similar behavior and suffer the same deficiencies. Quantization lobes occur in the limited field of view array because the subarrays are arranged periodically in the array. At broadside (and for a uniform array), the array appears continuous and no extraneous lobes are radiated. When the array is scanned by the phase shifters behind the subarrays, the aperture phase is quantized because the subarrays themselves have a pattern that radiates broadside. The array factor of this array of subarrays is, however, scanned to the new chosen scan angle, giving rise to quantization lobes that are only suppressed at broadside.

An upper bound for LFOV scanning in one dimension is that for idealized subarrays with spacings of dimension $D$, and maximum scan angle $\theta_{0}$, the subarray size $D$ is given by the following relationship:

$$
\left(\frac{D}{\lambda_{0}}\right) \sin \left|\theta_{0}\right| \leq 0.5 \text {. }
$$

This limit is reached using an idealized subarray pattern that is pulse-shaped, with a flat top over that range [2].

For LFOV, therefore, the quantization lobes occur because the subarray pattern is stationary, while the array factor is scanned. However, in the case of using time delays for broadband radiation, the phase shifters in each subarray do scan the subarray patterns correctly at center frequency, and the time-delayed array of subarrays remains at the chosen scan angle for all frequencies. However, away from center frequency, the phase-steered subarray patterns do not scan to the chosen location $u_{0}$; but "squint" to the new location $u_{0}\left(f_{0} / f\right)$ for $u_{0}=\sin \theta_{0}$. Since the subarray patterns and the array factor peaks are no longer aligned, this case also radiates quantization lobes.
This usage is indicated on the right side of Figure 1, where it shows a subarray with element level phase shifters and time delay at the subarray input. For modest bandwidth, an upper bound on the system bandwidth in this case is again obtained by the idealized case in which the subarray spacing $D$ is limited by the following equation:

$$
\frac{\Delta f}{f_{0}}\left(\frac{D}{\lambda_{0}}\right) \sin \left|\theta_{0}\right| \leq 1.0 .
$$

Reaching this limit is again only achieved if the subarray is designed to produce the "ideal" pulse-shaped pattern.

Throughout this paper, the data that are presented pertain to the wideband scanning case. It should be understood that the results apply equally well to the LFOV case. The LFOV case results are readily inferred from (1) and (2) resulting in the relationship of (3) for scan to some maximum angle $\theta_{\max }[3]$ :

$$
\frac{\Delta f}{f_{0}} \sin \left(\left|\theta_{0}\right|\right)=2 \sin \left(\left|\theta_{\max }\right|\right) .
$$

We have not discussed the issue of impedance matching elements over such wide bandwidths. The element lattice periodicity requires element spacing on the order of $0.5 \lambda$ at the highest frequency, and this makes them very closely spaced at the lowest frequency. This close spacing actually improves the array wideband impedance match, as discussed in the text by Munk [4]. Vivaldi elements, connected dipole arrays and matched microstrip patch elements, have all been used in wideband arrays.

\section{Subarray Technology for Space Applications}

The several upper bound equations noted above make it clear that there can be completely different applications for various subarray sizes. In the LFOV case, the subarray sizes can vary from about 3 wavelengths for \pm 10 degrees of scan to about 14 wavelengths for \pm 2 degrees of scan, and even larger for less scan. The wide instantaneous bandwidth is required for lower orbiting radars and communication arrays, where scanning requirements can exceed $\pm 20^{\circ}$ and array sizes up to hundreds of thousands of elements. Subarrays used to insert such time delay can be very large.

The technology for producing large subarrays with the qualities to suppress these quantization lobes depends upon the size of the subarray. Large subarrays have been constructed by dividing the array into an irregular group of elements and building special power divider networks for each subarray [5], or by using so-called dual-transform or overlapped subarray networks $[6,7]$. These networks use a multiple beam feed and a focusing lens to form overlapping distributions to radiate pulse-shaped subarrays that approximate the ideal flat-topped subarray radiation pattern. Such large subarrays formed in this manner can vary from 10 to perhaps 50 elements on a side and so are useful for very limited scan coverage or very narrow bandwidth scanned performance, as dictated by (1) and (2). Their advantage, however, is the huge savings in phase or time delay control devices and resulting cost savings. 
Small subarrays have been fabricated to produce overlap and an approximation to the pulse-shaped radiation patterns that have wider but still limited field of view $[8,9]$, or to applications involving very wide band radiation. Other technologies applicable to smaller subarrays include using simple rectangular subarrays but rotating them [10] or randomly spacing them to suppress the quantization lobes to use different-sized rectangular subarrays that do fill the aperture [11-13], or to use some sort of irregular subarrays that fill the aperture [14-16]. Of these, the randomly spaced subarrays produce excellent suppression of extraneous lobes, but radiate with reduced gain in proportion to the reduced filling of the aperture. The use of unequal size rectangular subarrays or dissimilar irregular subarrays introduces problems in excitation of the subarrays because of the need to use different power divider combinations within the array. The proposed method that we introduce employs only one type of irregular subarray within the array, and these are rotated (or rotated and flipped) to completely fill the array. The result is to form an array with non-periodically spaced subarray phase centers but with a tiled (filled) aperture that has full array gain.

\section{Irregular Polyomino Subarrays}

Figure 2 shows an array with subarrays and indicates the array filling with L-octomino subarrays. It also shows four rotations of an L-tetromino and two kinds of octominos, an L-octomino and a "point-up" octomino, that we use to fill the array. In this paper, we are concerned only with shapes that completely fill, or "tile" the array area, because the fully filled area has maximum aperture gain. There are many other polyomino shapes and sizes that can be used for tiling an array, and the branch of mathematics that is closest to polyomino tilings is called combinatorics, of which polyominos are a subset.

A more basic combinatoric problem may query whether a collection of identical polyominos could be tiled as to exactly fill a rectangle. Such collections are called rectifiable. One researcher, Michael Reid [17], has a compact, permanent link for the subject [18]. A study limited to octominoes will yield, in a recognized Wolfram reference [19], a list of 369 distinct octominos. These few topics are evidence of a concentrated and vibrant area of mathematical research.

Throughout this work, we have dealt exclusively with polyominos that fully tile the apertures but have allowed some or many polyominos to extend beyond the boundaries of the aperture, assuming that those squares outside of the aperture need not be excited.

Figure 3 shows a group of three-dimensional patterns radiated by an array of $64 \times 64$ elements at $f / f_{0}=1.3$ and $u_{0}=v_{0}=0.5$, where $u_{0}=\sin \theta_{0} \cos \phi_{0}, v_{0}=\sin \theta_{0} \cos \phi_{0}$, and $\theta_{0}$, and $\phi_{0}$ define the direction of scan. The array has a $40 \mathrm{~dB}$ Taylor taper, and therefore has low side lobes at broadside and/or center frequency. All element patterns are assumed to have a $\cos \theta$ angular dependence. The left pattern in Figure 3 is that of an array with time delay at every element. This pattern exhibits the design sidelobes of $-40 \mathrm{~dB}$ with a pattern gain of $37.3 \mathrm{~dB}$ as determined by integration. The center pattern corresponds to an array in which the individual elements are grouped into 512 rectangular subarrays (two elements in the elevation plane and four in the azimuth plane). This pattern has a gain of $37.03 \mathrm{~dB}$ (approximately a $0.3 \mathrm{~dB}$ loss due to phase error compared to the left pattern) and has five quantization lobes. The largest of these lobes is $11.45 \mathrm{~dB}$ below the gain at broadside. The pattern at right is produced by an array of $512 \mathrm{~L}$-octominos and radiates a main beam with a $36.83 \mathrm{~dB}$ gain (only $0.2 \mathrm{~dB}$ less than the array of rectangular subarrays). This pattern has no quantization lobes and has side lobes that are all at least $26.6 \mathrm{~dB}$ below the broadside gain.

These data are typical of results that have been presented previously to describe this work. In general, the various polyomino-based arrays have been found to have nearly the same gain but much smaller sidelobes than arrays of periodic, rectangular subarrays. Note that these residual side lobes are not suppressed quantization lobes, since the quantization lobe size remains constant relative to the main beam as array size is changed. Rather, we see that the residual side lobes of the polyomino-based arrays are reduced as the inverse of the array area. Our experience with arrays of up to 128 elements on a side indicate that the peak sidelobes continue to decrease by 5 to $6 \mathrm{~dB}$ with each quadrupling of array area.

Figure 4 addresses the question as to why the polyominobased arrays are successful at eliminating the quantization lobes. Clearly the procedure breaks up the periodicity of the array, and so one would expect a better result than with contiguous rectangular subarrays, but with this figure we investigate much suppression due entirely to the 


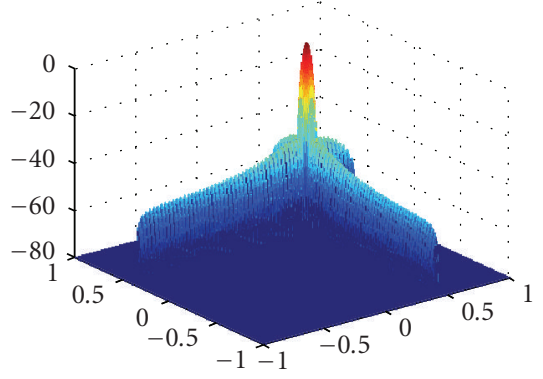

(a)

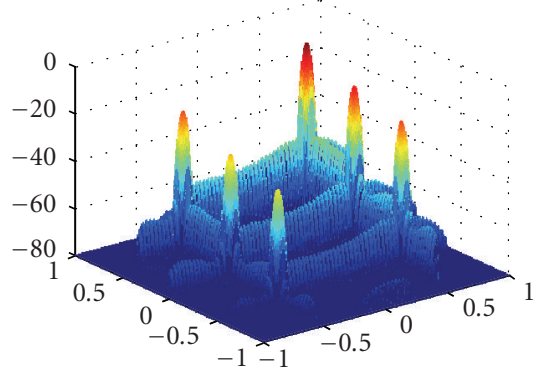

(b)

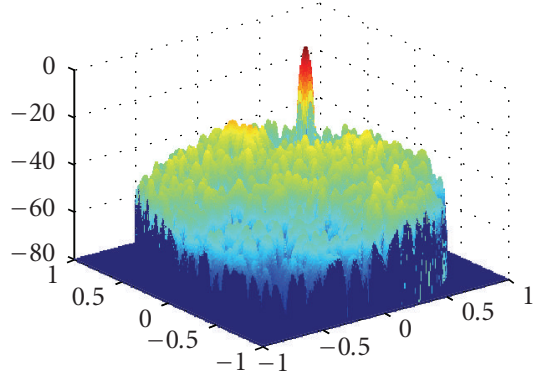

(c)

FIgURE 3: Radiation patterns at $f / f_{0}=1.3$ for an array with time delay at element level (a), at rectangular subarray inputs (b) and at L-octomino subarray inputs (c).

quasi-randomness and how much due to the changes in the subarray pattern amplitude as each is rotated.

Figure 4(a) shows an array of rectangular subarrays and the final pattern for an array of rectangular subarrays with $f / f_{0}=1.3$ and scanned to $u_{0}=v_{0}=0.5$. The elements are $0.5 \lambda$ apart in both planes, and the inter-subarray spacing of the eight element subarrays is $\lambda$ in azimuth and $2 \lambda$ in elevation. The array has 18 elements in azimuth and 24 in elevation. The contour levels are set at $\mathrm{dB}$ values of $-3,-10,-15$, and -20 . The scanned pattern, shown at right in Figure 4(a), has a set of 5 quantization lobes, with varying degrees of suppression due to the subarray pattern. Figure 4(b) shows the array factor for the array with the subarray patterns assumed omnidirectional, as illustrated on the left of Figure 4(b). All the quantization lobes of Figure 4(a) are present at the same locations, but are now grating lobes unattenuated by the subarray pattern. A comparison of the numerical data shows that the resulting array factor of Figure 4(a) has its grating lobes suppressed at least $12 \mathrm{~dB}$ by the subarray pattern. These peak quantization lobes are at the same levels for much larger arrays.

Figures 4(c) and 4(d) present the same situations as Figures 4(a) and 4(b), but for the case of irregular (point-up) subarrays Figure 4(c) shows an actual subarray configuration of "point-up" polyominos and the pattern for that array. Although the array is small, the figure again demonstrates significantly lower sidelobes than the array of rectangular subarrays Figure 4(a). All of the larger sidelobes are in the neighborhood of $-20 \mathrm{~dB}$, or $8 \mathrm{~dB}$ below those of the rectangular subarrays, and they are no longer located at the quantization lobe angles. The pattern shows that no quantization lobes remain. Figure 4(d) shows the array factor of the polyomino based array with the subarray patterns replaced by omnidirectional one. This pattern thus includes only the effect of the aperiodic grid of phase centers. This pattern shows that only one peak sidelobe remains at the quantization lobe locations shown in 4(b), while other peaks do not correspond to the quantization lobe locations. Note that some of these peaks are greater than $-10 \mathrm{~dB}$ since there are no element patterns to suppress them. The peak sidelobes now are distributed throughout a much wider area of space and our experience has shown that unlike the data of Figure 4(a), they continue to decrease with array size. Comparing Figures 4(a) and 4(c) shows that the combination of the rotating subarray patterns and the phase center displacement produce a much larger role in sidelobe suppression than phase center displacement alone, but the phase center displacement is the major contributor to the successful result.

\section{Power Dividers for Tetrominos and Octominos}

In general, one would expect that assembling an array from irregular subarrays would entail some extra burden in fabrication or assembly. However, there is little added assembly cost for an array of polyominos that are all identical but rotated in the plane. Therefore we have chosen to use polyominos that have $2^{n}$ elements for $n=2$, and 3 for this paper, so that the subarrays can be excited by lossless power dividers. Other polyominos of larger size " $n$ " can also be used, but larger polyominos have less bandwidth (or for LFOV they have a more limited scan region), and we have not investigated these limits. Very few larger polyominos can actually tile rectangular areas without leaving voids in the surface, and it seems that with the exception of rectangular shapes one would need to use a multiplicity of higher-order polyominos to properly tile a rectangular surface. The array elements themselves are on a fully periodic grid and so the aperture is unchanged from conventional apertures. Only the feed matrix is constrained to introduce the aperiodicity needed to eliminate quantization lobes.

Figures 5, 6, and 7 show the power dividers and feed points (black rectangles) and a section of the filled arrays corresponding to the shapes noted in Figure 2 and investigated in this paper. Notice that all power dividers in any figure are identical, but because of the rotation the excitation points form an irregular pattern.

\section{Peak Sidelobe Behavior of Tetrominos and Octominos}

Peak sidelobes are not readily predicted analytically for arrays of irregular subarrays and so numerical results have been used to relate them to the rectangular subarray case. The peak 

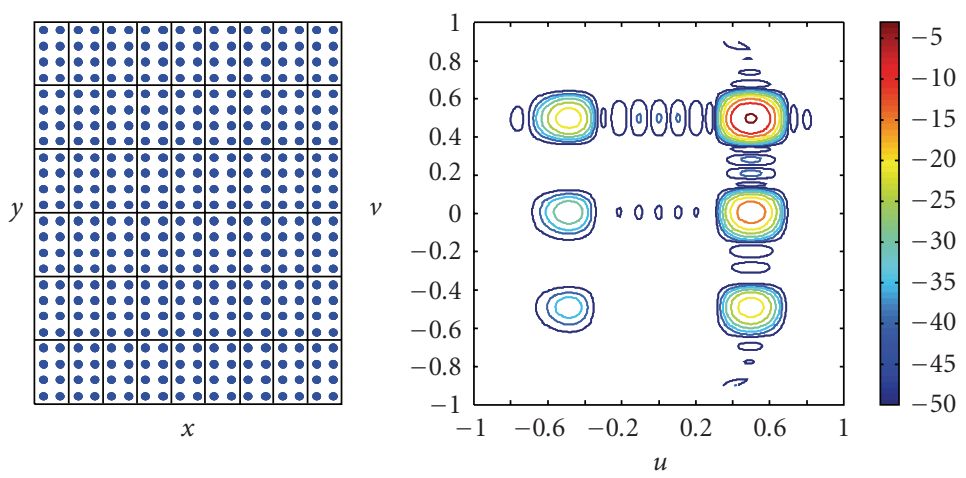

(a) Array of rectangular subarrays
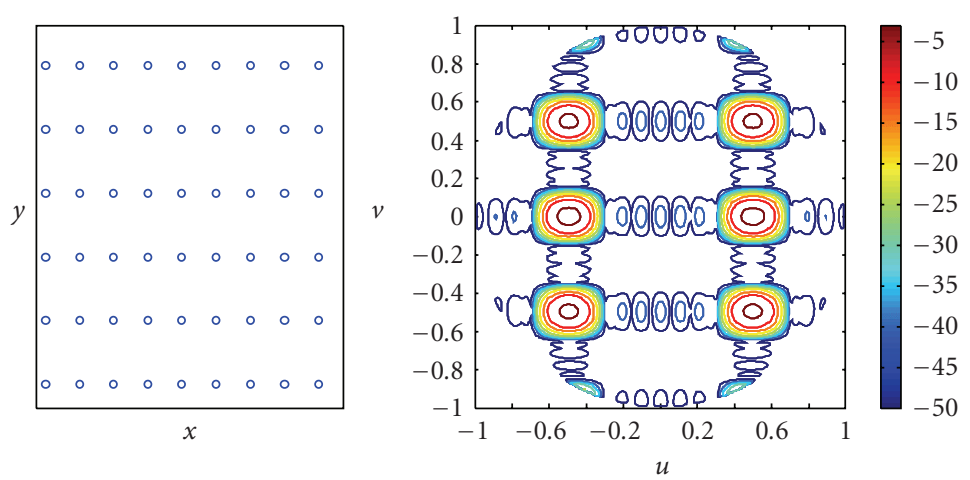

(b) Array of omnidirectional elements at rectangular subarray phase centers
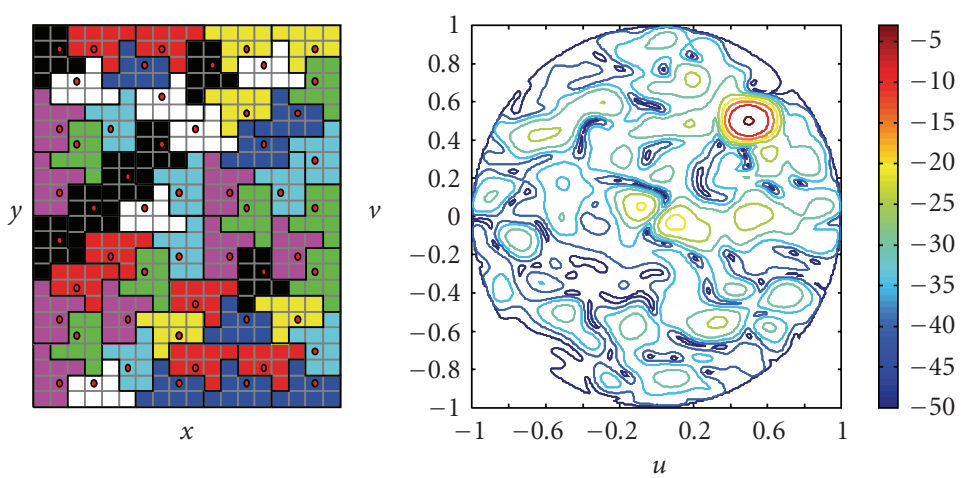

(c) Array of octomino subarrays. Subarray phase centers are indicated by red dots
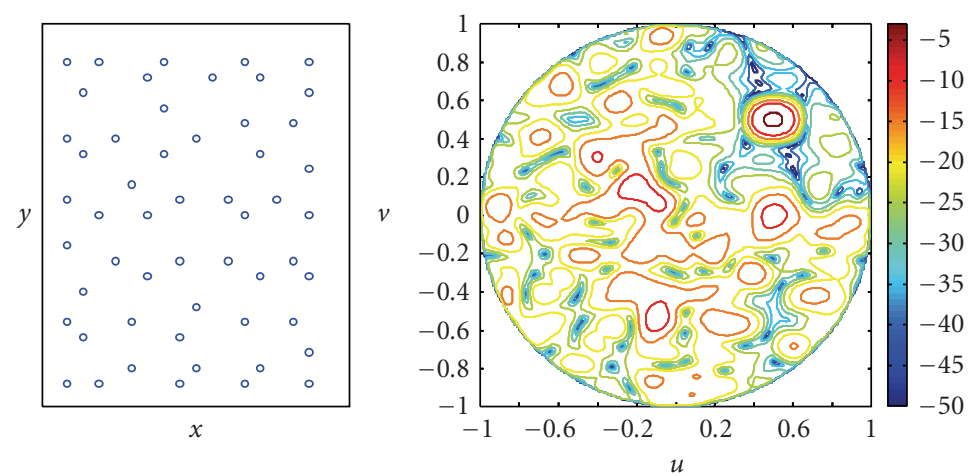

(d) Array of omnidirectional elements at octomino subarray phase centers

FIGURE 4: Sidelobe structure for $18 \times 24$ element array of 54 subarrays of rectangular or L-octomino subarrays. 


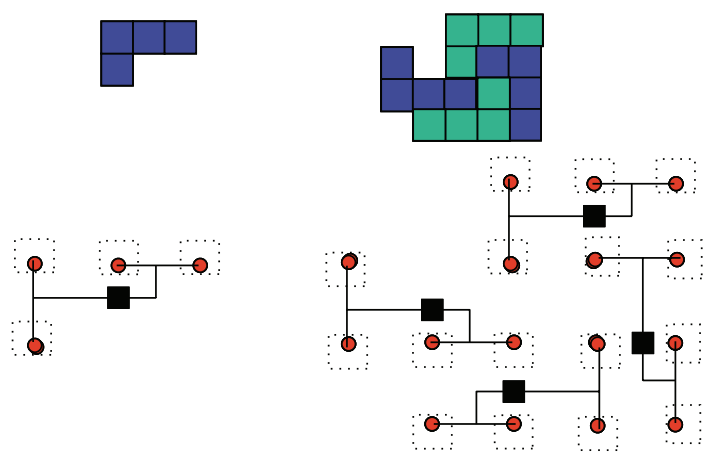

FIGURE 5: L-tetromino power divider and array section.

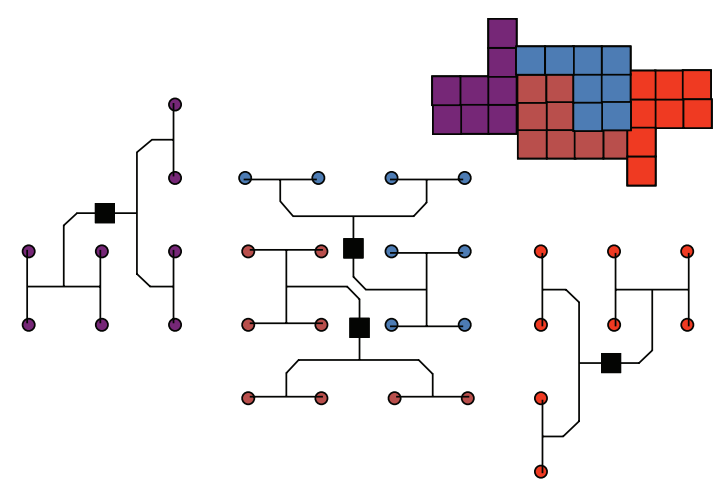

FIgURE 6: L-octomino power divider and array section.

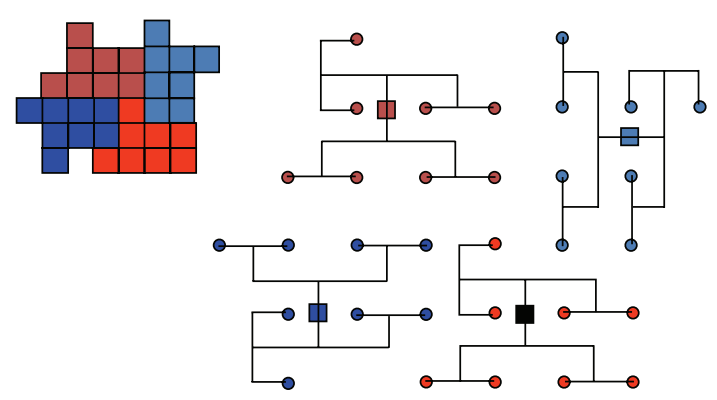

FIgURE 7: Point-up power divider and array section.

sidelobes depend upon scan angle and, for the wideband case, frequency offset ratio " $r$ ", but once these parameters are chosen, the peak sidelobes decrease monotonically with array size and very nearly like a constant times $1 / N$ for a large $N$ element planar array. The array gain increases with array area modified by the phase error introduced within each subarray.

The bandwidth of polyomino subarrays is primarily determined by gain falloff with scan, since there are no quantization lobes, and the peak sidelobes continue to decrease monotonically with array size. At the highestfrequency the element spacing is chosen to be spaced a half wavelength apart to avoid the element level grating lobes.

Figure 8 shows five sets of gain curves for square arrays as the array size is varied. These, and all other gain curves shown in this paper, were computed by integrating the array pattern using an assumed $\cos \theta$ element pattern. The figure

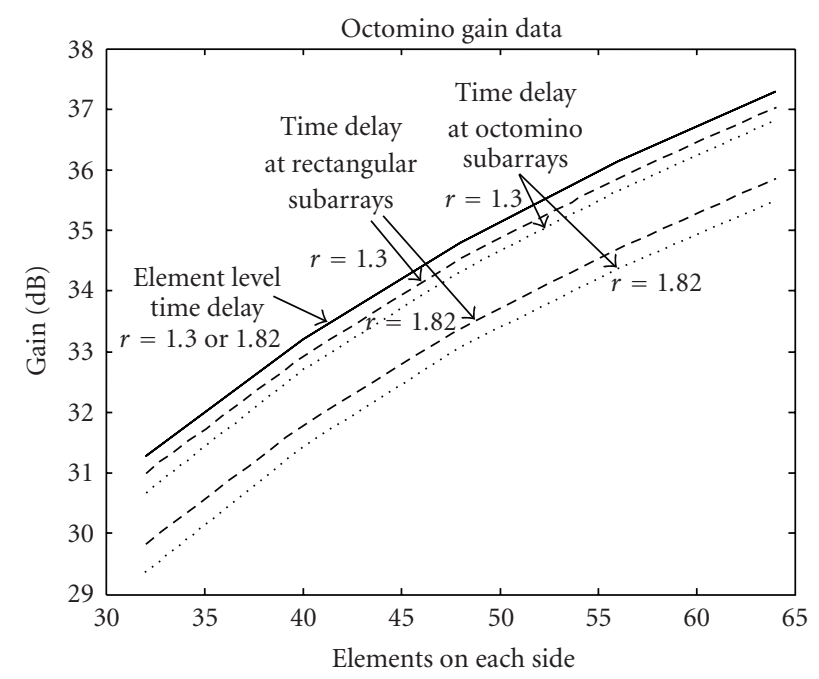

FIGURE 8: Gain comparison of arrays with time delay at element levels versus time delay at rectangular and L-octomino subarrays $r$ $=f / f_{0}=1.3$ and 1.82 .

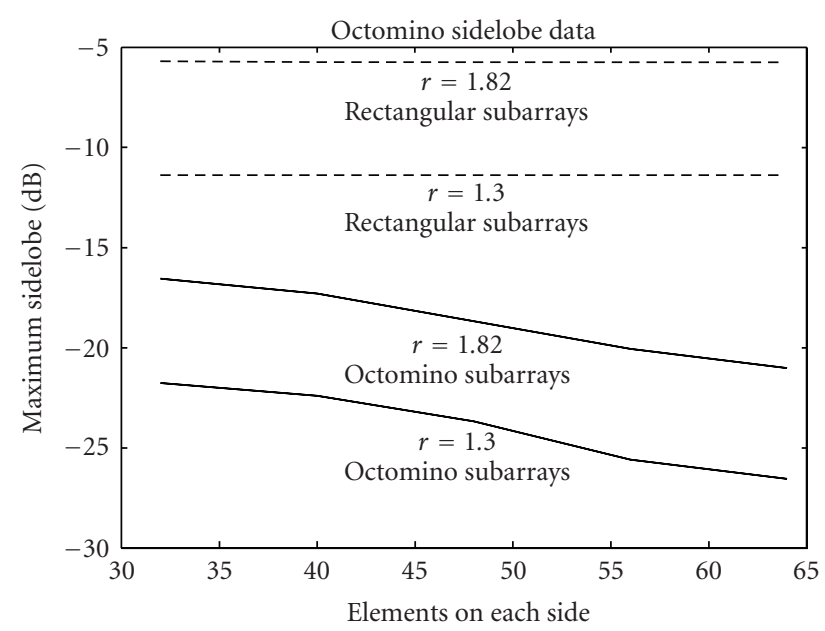

Figure 9: Peak sidelobe level of rectangular and L-octomino subarrays $r=f / f_{0}=1.3$ (moderate bandwidth) and $r=1.82$ (wide band).

compares the gain of arrays with time delay at the subarray level for rectangular or octomino subarrays. The gain of arrays with time delay at the element level is given as the solid curve. This represents the ideal array gain and is higher than any subarrayed case because there is no phase error.

The several time-delayed subarray cases plotted include one with about $2: 1$ bandwidth $\left(r=f / f_{0}=1.3\right)$ for the upperband limit, and one with about 10:1 bandwidth $(r=1.82$ upper-frequency) and the design low-frequency of $r=0.182$ (lower-frequency band not shown). We have not shown the lower-frequency results for this ultra wideband case because at this lower limit the subarray spacing is so small that there are no quantization lobes in real space. Unlike the narrower band situations where there are quantization lobes at both high and low-frequency extremes, here the subarray imposes no low-frequency limit; only the element spacing 


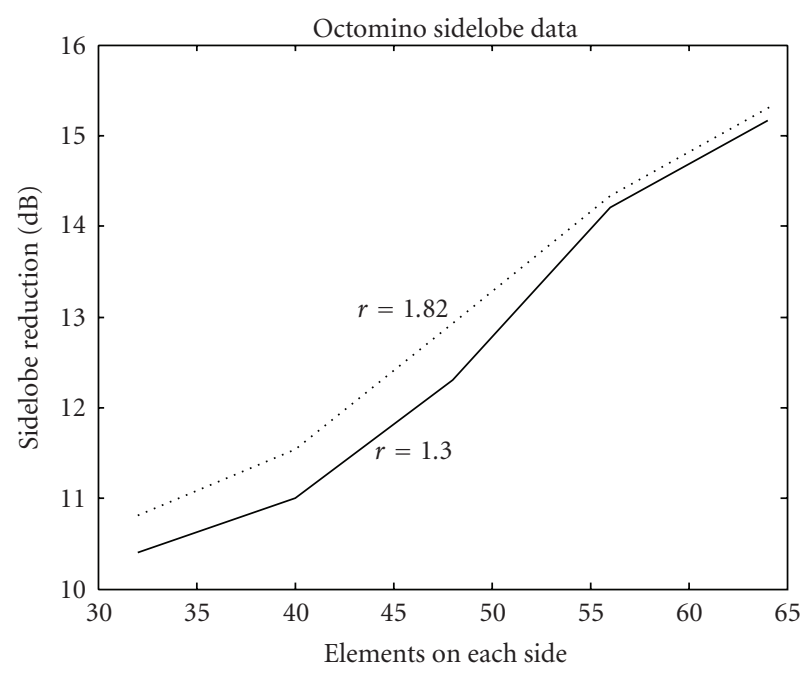

FIGURE 10: Sidelobe reduction factor (max rectangular subarray quantization lobe level-largest octomino sidelobe level) $\mathrm{dB}$ for moderate and wide band signals.

and impedance considerations provide any low-frequency limitation. In the cases discussed, the low-frequency could be chosen as low as the element design would allow, with no limitation imposed by the subarraying technique.

The curves shown in Figure 8 include the solid curve with full element level time delay and four curves with time delay at the input port to each subarray. The dashed curves are for rectangular subarrays $(2 \times 4$ elements $)$, one with highfrequency $r=1.3$ (bandwidths $r=f / f_{0}=0.7$ to 1.3 ) and one with much higher bandwidth $\left(r=f / f_{0}=0.182\right.$ to 1.82 , a $10: 1$ bandwidth).

The solid curve at the top of the figure is the gain using time delay at each element. These integrated data are almost exactly equal to the usual large array approximation for an aperture with aperture efficiency $\varepsilon_{a}$ :

$$
G=4 \pi \frac{A}{\lambda^{2}} \varepsilon_{a} \cos \theta
$$

Notice that this single curve is the array gain for both frequency offsets; $r=1.3$ and 1.82 , because the element spacing is set to $0.5 \lambda$ at each frequency, so the array size is the same in normalized terms and the gain is also the same. The curves for time delay at the subarray input ports are shown dashed for rectangular subarrays and are approximately $0.27 \mathrm{~dB}$ below the time delayed array data at $r=1.3$ and $1.45 \mathrm{~dB}$ below the curve at $r=1.82$. The added loss is caused by the incremental phase error across the 8element subarrays. The two dotted curves of Figure 8 give the corresponding gain for the L-octomino subarray based arrays, and lie approximately $0.2 \mathrm{~dB}$ below the curves for the rectangular subarrays, owing to the slightly greater phase error in the polyomino subarrays.

Figure 9 shows the corresponding peak sidelobe levels for the subarrayed cases of Figure 8. The peak sidelobe level for the element level time delayed array is not shown because it is just the idealized Taylor pattern with $-40 \mathrm{~dB}$ peak sidelobes.

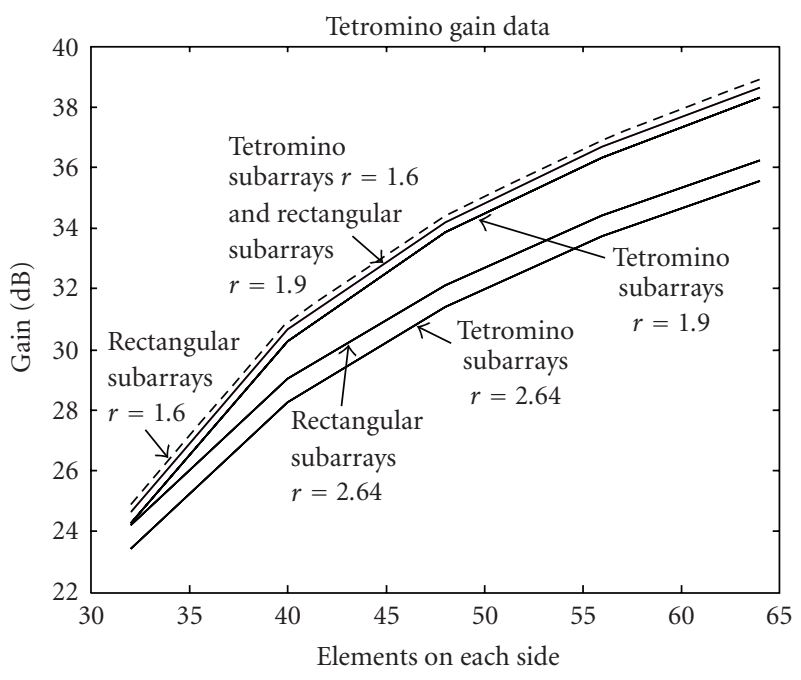

Figure 11: Gain comparison of arrays with time delay at element levels versus time delay at rectangular and L-tetromino subarrays $r$ $=f / f_{0}=1.6, r=1.9$ and $r=2.64$ (wide band).

In this Figure the dashed patterns give the peak sidelobe level of arrays of rectangular $(2 \times 4)$ subarrays and show constant levels of $11.4 \mathrm{~dB}$ for $r=1.3$, and $-5.75 \mathrm{~dB}$ below the broadside gain (or only $4.23 \mathrm{~dB}$ below the projected area gain at $\left.u_{0}=v_{0}=0.50\right)$ for $r=1.82$. The quantization lobe level is independent of array size because the lobe samples the subarray pattern at the same point for any size array. The two solid curves in Figure 9 are the peak sidelobes for arrays of L-octomino subarrays for the two frequencies noted. Note that the improvement in comparison with the rectangular subarray results is nearly the same at both frequencies. Figure 10 shows this added sidelobe reduction to vary from about $10.5 \mathrm{~dB}$ for arrays 32 elements on a side to about $15.5 \mathrm{~dB}$ for 64 element arrays, but more interestingly this sidelobe reduction is almost the same whether the rectangular subarray peak sidelobes are $-11.4 \mathrm{~dB}$ (for $r=1.3$ ) or -5.75 (for the $r=1.82$ ). This indicates that these limited results can be expected to be useful for estimating behavior of the arrays at other frequencies. The rectangular subarray data of Figure 9 are very simple to compute, but using the result of Figure 10, one can obtain a good estimate of peak sidelobe level for nearly any frequency range that one would want to estimate. In addition, we have found it a general rule for all the cases we have tested, that quadrupling the array size results in at least a $5 \mathrm{~dB}$ increase in this sidelobe reduction factor, so one can extrapolate these data to get results for various sizes as well as various frequencies.

Figures 11-13 show similar results for L-tetrominos when these are operated over about twice the bandwidth. The normalized upper frequency ranged from $r=1.6$ to 2.64. Figure 11 shows the gain of an array of the 4 element subarrays, comparing rectangular (square) $2 \times 2$ subarrays with the L-tetromino subarrays. The difference in gain between the four element rectangular subarrays and the Ltetromino subarrays is about $0.2 \mathrm{~dB}$ maximum at the $r=$ 1.6 frequency limit, and about $0.8 \mathrm{~dB}$ at the higher $r=2.64$ frequency limit. Figure 12 shows the sidelobe behavior of 


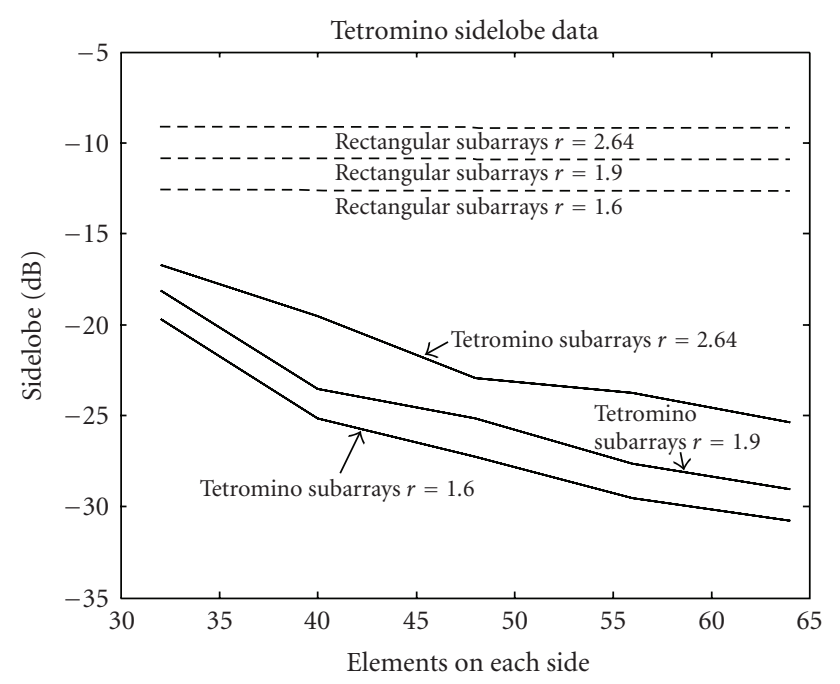

Figure 12: Peak sidelobe level of rectangular and L-tetromino subarrays $r=f / f_{0}=1.6$ (moderate bandwidth), $r=1.9$ and $r=2.64$ (wide band).

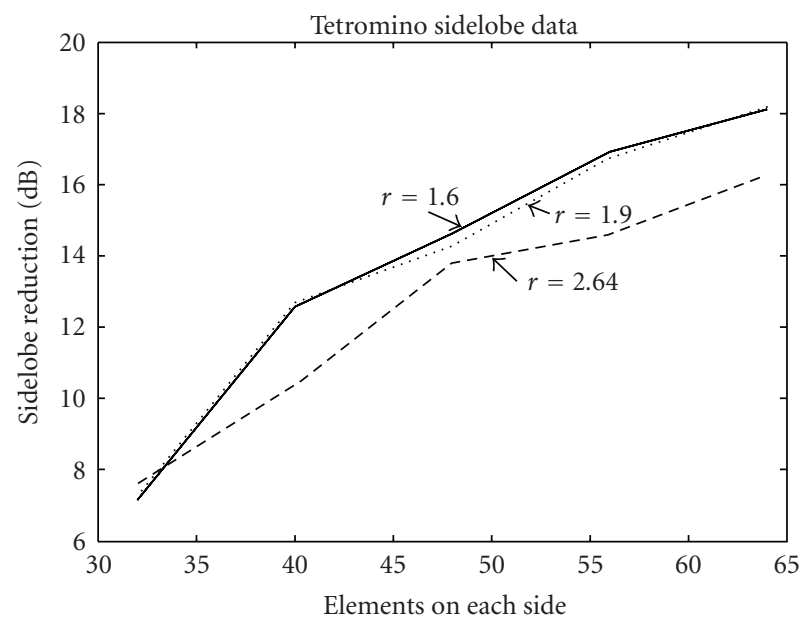

FIGURE 13: Sidelobe reduction factor (max rectangular subarray quantization lobe level-largest octomino sidelobe level) $\mathrm{dB}$ for moderate and wide band signals.

the arrays at both frequencies, and Figure 13 compares the sidelobe reduction for these cases. Here the reduction varies over a wider range than the octomino case but the slope of the curve is approximately the same for larger arrays. Even for the wideband $r=2.64$ case the sidelobe reduction is within $2 \mathrm{~dB}$ of the lower bandwidth cases, again indicating the utility of these curves in generalizing to other frequencies and array sizes.

\section{Conclusion}

This paper has presented new results showing the application of polyomino-shaped subarrays to large space-based arrays that scan over a limited field of view with modest bandwidth, or over wider bandwidth and wide scan angles. In both cases, subarray technology may be necessary to reduce cost by decreasing the number of phase shifters or time delay devices.

The paper compares the use of irregular polyominos versus the corresponding rectangular subarrays and shows that with only few tenths of a $\mathrm{dB}$ gain reduction the 8element polyomino subarrays can reduce the peak sidelobes by more than $15 \mathrm{~dB}$ for $64 \times 64$ element arrays. Potentially much lower sidelobes can be obtained for larger arrays since sidelobe reduction improves by $5-6 \mathrm{~dB}$ for each quadrupling of array size. Results for smaller (tetromino) subarrays are in accord and provide even wider bandwidth.

Finally, it is shown that this sidelobe reduction is primarily a function of array size, not the frequency of operation. This leads to the useful conclusion that by focusing attention on the sidelobe reduction itself, it is possible to estimate peak sidelobe levels for tetromino and octomino arrays directly from the available rectangular subarray data.

\section{Acknowledgments}

This work was supported in part by the Air Force Office of Scientific Research, under Dr. A. Nachman, and in part by the Center for Advanced Sensor and Communications Antennas, Department of Electrical and Computer Engineering, University of Massachusetts, Amherst, Mass, USA. The work was conducted at the Sensors Directorate, Air Force Research Laboratory, Hanscom AFB, Mass, USA.

\section{References}

[1] R. Tang, "Survey of time delayed beam steering techniques," in Phased Array Antennas: Proceedings of the 1970 Phased Array Antenna Symposium, pp. 254-260, Artech House, Dedham, Mass, USA, 1972.

[2] R. J. Mailloux, Electronically Scanned Arrays, Morgan \& Claypool, San Rafael, Calif, USA, 2007.

[3] R. J. Mailloux, "Subarray technology for large scanning arrays," in Proceedings of the 2nd European Conference on Antennas and Propagation (EuCAP '07), pp. 1-6, Edinburgh, UK, November 2007.

[4] B. A. Munk, Finite Antenna Arrays and FSS, Wiley-IEEE Press, New York, NY, USA, 2003.

[5] U. R. O. Nickel, "Monopulse estimation with subarray output adaptive beamforming and low sidelobe sum and difference beams," in Proceedings of IEEE International Symposium on Phased Array Systems and Technology, pp. 283-288, Boston, Mass, USA, October 1996.

[6] S. P. Skobelev, "Methods of constructing optimum phasedarray antennas for limited field of view," IEEE Antennas and Propagation Magazine, vol. 40, no. 2, pp. 39-49, 1998.

[7] J. S. Ajioka and J. L. McFarland, "Beamforming feeds," in Antenna Handbook: Theory, Applications and Design, Y. T. Lo and S. W. Lee, Eds., chapter 19, Van Nostrand Reinhold, New York, NY, USA, 1988.

[8] J. S. Herd, S. M. Duffy, and H. Steyskal, "Design considerations and results for an overlapped subarray radar antenna," in Proceedings of IEEE Aerospace Conference (AERO '05), pp. 1087-1092, Big Sky, Mont, USA, March 2005.

[9] S. M. Duffy, D. D. Santiago, and J. S. Herd, "Design of overlapped subarrays using an RFIC beamformer," in Proceedings 
of IEEE Antennas and Propagation International Symposium (AP-S '07), pp. 1949-1952, Honolulu, Hawaii, USA, June 2007.

[10] P. Russo, "Antenna which assures high speed data rate transmission links between satellites and between satellites and ground stations," US patent no. 5262790, November 1993.

[11] R. L. Haupt, "Optimized weighting of uniform subarrays of unequal sizes," IEEE Transactions on Antennas and Propagation, vol. 55, no. 4, pp. 1207-1210, 2007.

[12] H. Wang, D.-G. Fang, and Y. L. Chow, "Grating lobe reduction in a phased array of limited scanning," IEEE Transactions on Antennas and Propagation, vol. 56, no. 6, pp. 1581-1586, 2008.

[13] P. Rocca, L. Manica, M. Benedetti, R. Azaro, and A. Massa, "Simultaneous optimization of subarray weights and sizes for low sidelobe synthesis of large array antennas," in Proceedings of IEEE International Symposium on Antennas and Propagation (APS '08), pp. 1-4, San Diego, Calif, USA, July 2008.

[14] V. Pierro, V. Galdi, G. Castaldi, I. M. Pinto, and L. B. Felsen, "Radiation properties of planar antenna arrays based on certain categories of aperiodic tilings," IEEE Transactions on Antennas and Propagation, vol. 53, no. 2, pp. 635-644, 2005.

[15] R. J. Mailloux, S. G. Santarelli, and T. M. Roberts, "Wideband arrays using irregular (polyomino) shaped subarrays," Electronics Letters, vol. 42, no. 18, pp. 1019-1020, 2006.

[16] K. C. Kerby and J. T. Bernhard, "Sidelobe level and wideband behavior of arrays of random subarrays," IEEE Transactions on Antennas and Propagation, vol. 54, no. 8, pp. 2253-2262, 2006.

[17] http://www.math.ucf.edu/ reid/Polyomino/index.html.

[18] http://tinyurl.com/qsrz8.

[19] http://mathworld.wolfram.com/Octomino.html. 

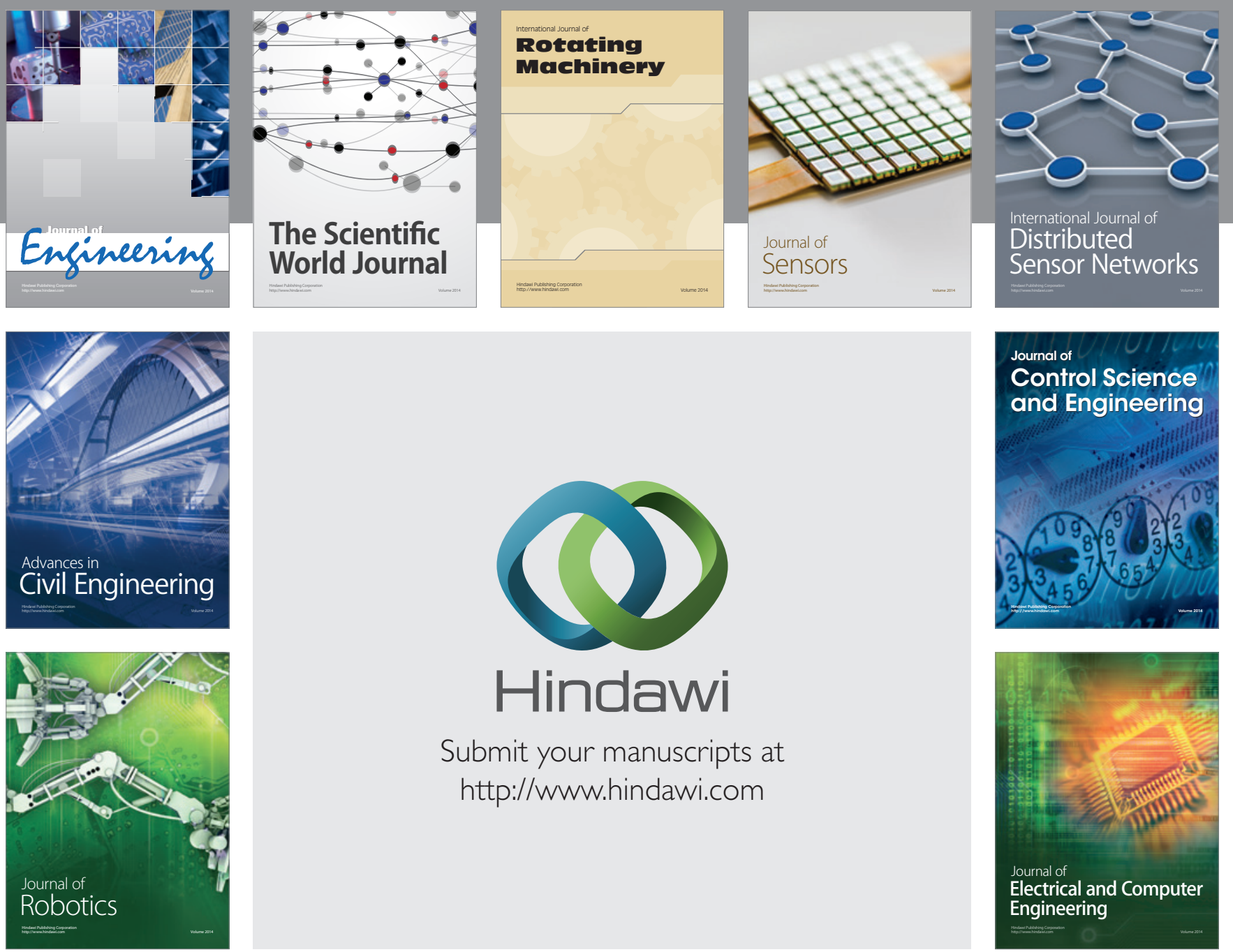

Submit your manuscripts at

http://www.hindawi.com
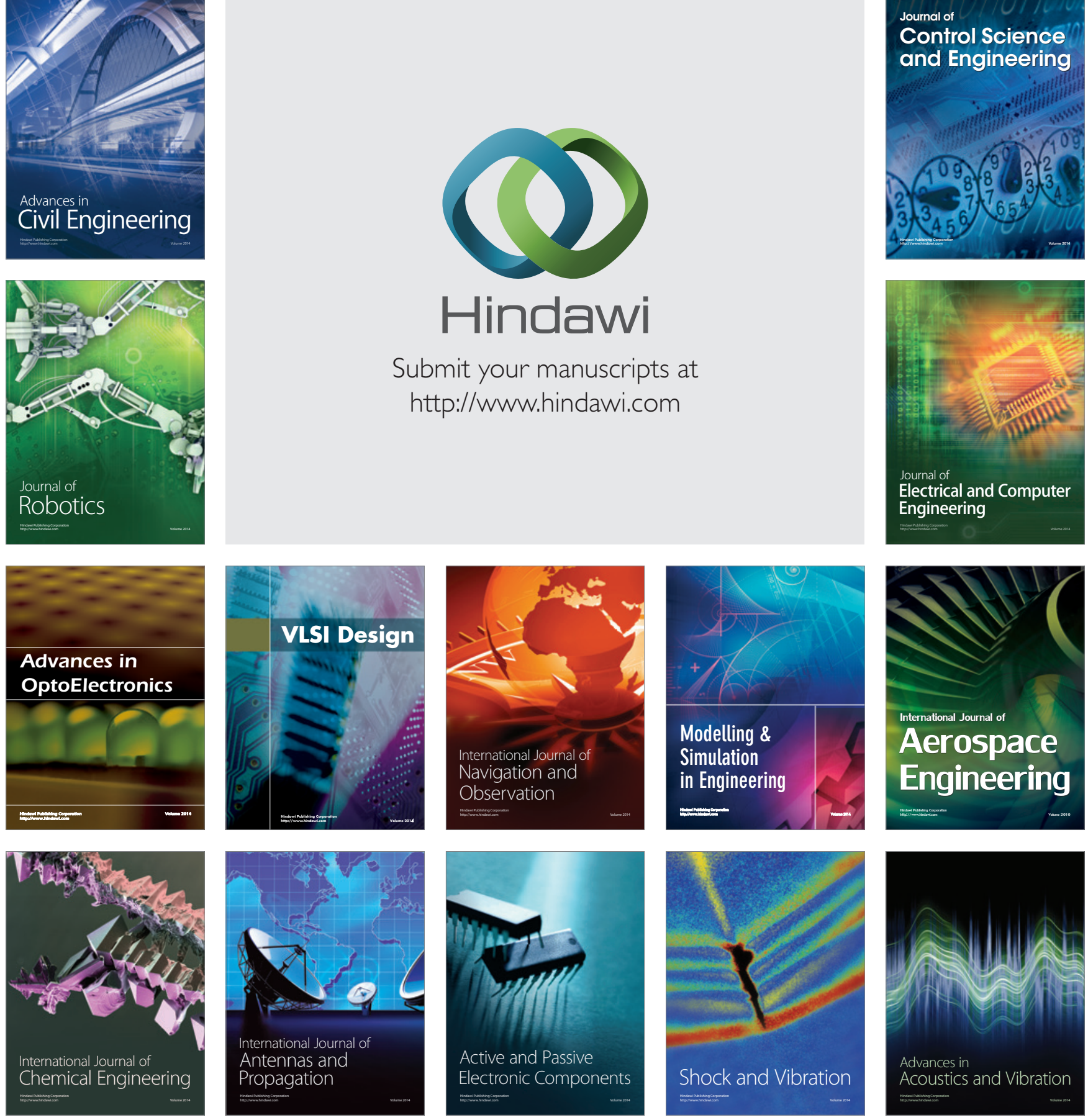